\title{
Control of critical coupling in a ring resonator-fiber configuration: application to wavelength-selective switching, modulation, amplification, and oscillation
}

\author{
John M. Choi, Reginald K. Lee, and Amnon Yariv \\ California Institute of Technology, M/S 136-93, 1200 East California Boulevard, Pasadena, California 91125
}

Received March 13, 2001

By controlling the internal loss of a ring resonator near critical coupling, we demonstrate control of the transmitted power in a fiber that is coupled to the resonator. We also demonstrate wavelength-selective optical amplification and oscillation. (C) 2001 Optical Society of America

OCIS codes: $060.2320,060.4080,060.1810,060.2330,060.4510,230.5750$.

The coupling of power between an optical waveguide and a ring resonator is a subject of continuing interest. $^{1-4}$ This interest intensified recently, due, in large measure, to a recognition of the potential application of this phenomenon is a variety of tasks important to optical fiber communication. These include ${ }^{5-7}$ modulation, switching, dispersion compensation, and as we will show in what follows, wavelength-selective amplification and oscillation.

The basic experimental configuration is shown in the inset of Fig. 1. A wave of (power-normalized) amplitude $a_{1}$ is incident upon the coupling region and exits as $b_{1}$. It was shown recently that the power-transfer characteristics $\left|b_{1} / a_{1}\right|^{2}$ of this configuration are described by a universal relation ${ }^{5}$ :

$$
\left|\frac{b_{1}}{a_{1}}\right|^{2}=\frac{\alpha^{2}+|t|^{2}-2 \alpha|t| \cos \theta}{1+\alpha^{2}|t|^{2}-2 \alpha|t| \cos \theta}
$$

where $t$ is the diagonal element of the coupling matrix relating the input and output fields at the coupling element

$$
\left|\begin{array}{l}
b_{1} \\
b_{2}
\end{array}\right|=\left|\begin{array}{cc}
t & \kappa \\
k^{*} & -t^{*}
\end{array}\right|\left|\begin{array}{c}
a_{1} \\
a_{2}
\end{array}\right|
$$

which in the lossless case obeys $|t|^{2}+|\kappa|^{2}=1 . \quad \alpha$ represents the circulation loss factor in the ring, according to $a_{2}=b_{2}[\alpha \exp (i \theta)]$. In passive resonators $\alpha<1$. In a lossless resonator $\alpha=1$. The inclusion of an optical amplifier in the ring can allow values of $\alpha>1$. $\theta=\beta L_{\text {loop }}$ is the circulation phase shift.

At resonance $\theta=m 2 \pi$ ( $m$ is an integer), and the transmission becomes

$$
\left|b_{1}\right|^{2}=\frac{(\alpha-|t|)^{2}}{(1-\alpha|t|)^{2}} .
$$

A plot of Eq. (3) is shown in Fig. 2. Two features of this figure illustrate most of the potential applications listed above: (1) There will exist a condition, $\alpha=|t|$, for which the transmitted power is zero. Following the microwave convention, in which this phenomenon is well known, ${ }^{8}$ we previously referred to this condition as critical coupling. ${ }^{5}$ At critical coupling the resonator mode loses the same power to the internal dissipation mechanisms (proportional to $1-\alpha^{2}$ ) as to the coupling to the outgoing fiber mode (proportional to $1-|t|^{2}$ ). (2) The second important feature is the steepness of the transmission characteristics at $\alpha>|t|$. It is apparent that in reasonably high- $Q$ resonators $(\alpha \approx 1)$ small modulation of $\alpha$, or $t$, can cause large modulation of the transmitted power. This can be used, for example, to construct electro-optic modulators with values of $V_{\pi}$ that are 2 orders of magnitude smaller than those of conventional Mach-Zehnder electrooptic modulators. It is interesting to note that, in the undercoupled region, $\alpha<t$, as the gain is increased, the power transmission decreases until the critical coupling point.

To exploit the critical coupling phenomenon profitably we need, according to Eq. (3), to be able to

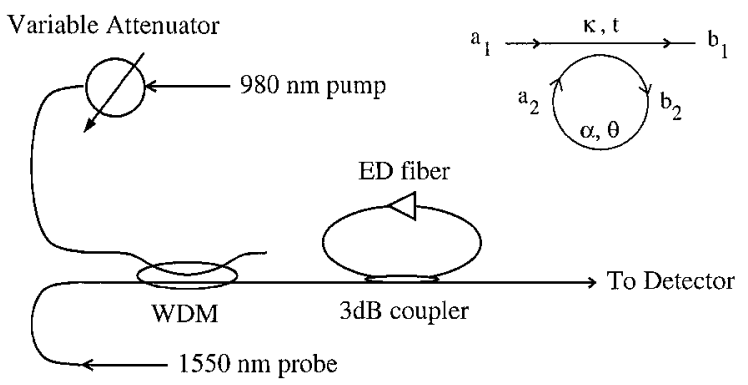

Fig. 1. Experimental setup used to measure the fiber-ring-waveguide system. A fused coupler is used as the coupling mechanism. Erbium-doped fiber (ED) is used in the ring to allow loss or gain modulation. A wavelength-division multiplexer (WDM) is used to add the 980-nm pump, allowing control of the coupling characteristics. Inset, conventions used in referring to the resonator-waveguide system. The input port is $a_{1}$, the output port is $b_{1}$, the loss in the resonator is $1-\alpha^{2}$, the coupling is $t$, and the round-trip phase delay from the resonator is $\theta$. From energy conservation, $|t|^{2}+|\kappa|^{2}=1$ and $a_{2}=\alpha \exp (i \theta) b_{2}$. 


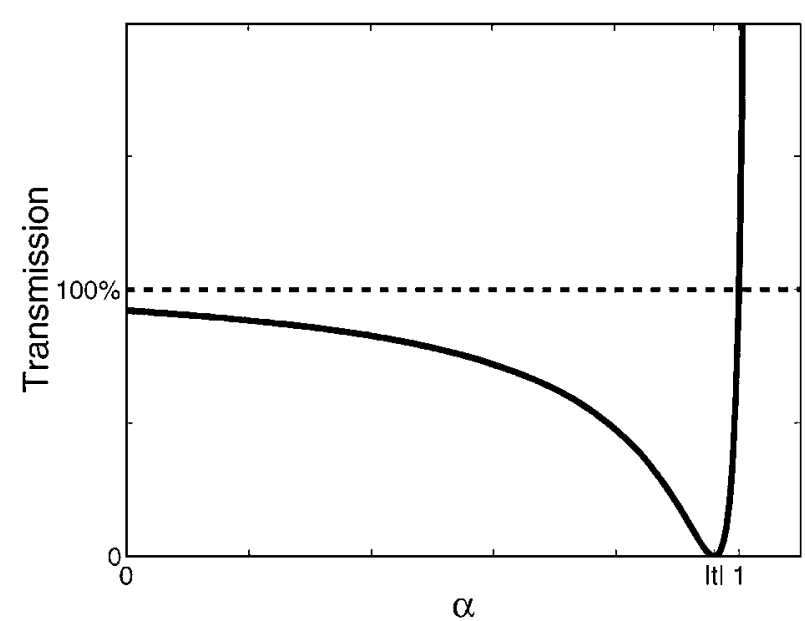

Fig. 2. Theoretical normalized power transmission as a function of internal cavity loss. The critically coupled point (zero transmission) occurs at $\alpha=|t|$. In the undercoupled region, $\alpha<t$, increasing the gain in the resonator up to the critically coupled condition decreases the power transmission.

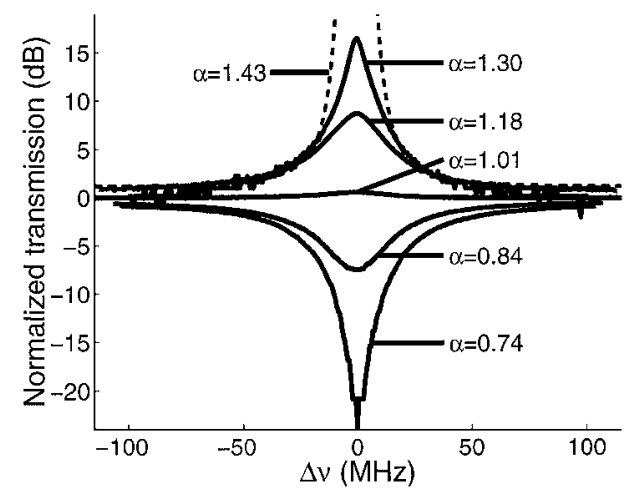

Fig. 3. Transmission spectrum of the ring resonatorwaveguide system. The curve with $\alpha=0.74$ when the transmission at resonance is $-22 \mathrm{~dB}$ is a state near critical coupling. Near-unity transparency $(\alpha=1.0)$ is observed when the $980-\mathrm{nm}$ pump power into the ring is approximately $6.8 \mathrm{dBm}$. Frequency-selective amplification near the resonance frequency is observed when $\alpha>1$. The dashed curve shows laser oscillation $(\alpha=1 /|t|)$, which was observed when the pump power coupled into the ring was $\sim 7.6 \mathrm{dBm}$.

control, or modulate, the coupling $t$ or the internal $\operatorname{loss} \alpha$. In this Letter we demonstrate the feasibility of controlling the internal loss parameter $\alpha$.

Our experimental arrangement is shown in Fig. 1. The coupling between the ring resonator and the fiber waveguide was provided by a fused $3-\mathrm{dB}$ coupler. Of the $59 \mathrm{~cm}$ of the circumference, $43 \mathrm{~cm}$ consisted of an erbium-doped fiber. The erbium-doped fiber was pumped by a 980-nm pump laser, and control of this pump power enabled us to control $\alpha$ over a large region straddling the critical, $\alpha=|t|$, point. We could also easily operate with $\alpha>1$.

Figure 3 shows experimental transmission plots as a function of the optical frequency deviation, with the loss parameter, $\alpha$, corresponding to a measured 980-nm optical pump power as a parameter. The loss parameter, $\alpha$, was calculated from the linewidth

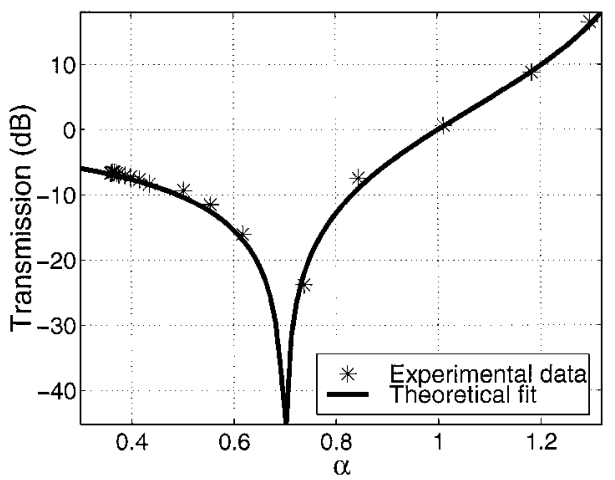

Fig. 4. Measurement of the transmission coefficient at resonance as a function of $\alpha$. Critical coupling (in this case a $-22-\mathrm{dB}$ transmission) as well as net gain is observed.

and depth of the transmission curve. This fit results in a very accurate measure of the loss that is due to the sensitivity of the transmission line shape to this parameter. We observe the existence of critical coupling (a -22-dB dip in transmission, ideally a zero dip) when $\alpha$ is 0.74 . The theoretical value of $\alpha$ at critical coupling is 0.707. Also apparent is a (near) unity $(\alpha=1)$ transmission transparency when the pump power coupled into the ring is $\sim 6.8 \mathrm{dBm}$ and the net optical gain at higher pump levels $(\alpha>1)$ reaches a peak gain of $15 \mathrm{~dB}$. Laser oscillation $(\alpha=1 /|t|)$ was observed when a pump power greater than $7.6 \mathrm{dBm}$ was coupled into the ring. The extrema of the transmission plots of Fig. 3 are plotted in Fig. 4 as a function of the loss parameter, $\alpha$. Figure 4 displays all the basic features of the theoretical plot of Fig. 2 and demonstrates the feasibility of controlling the transmitted power by electrical means.

The top two curves of Fig. 3 illustrate the possibility of wavelength-selective amplification of light propagating in the fiber, since only wavelengths corresponding to resonances of the ring resonator are amplified (or modulated), while other wavelengths pass through the ring unaffected. This feature allows the possibility of implementation in wavelengthdivision multiplexing systems. The device described here thus demonstrates some of the main functions that are important for communication: light generation and amplification, modulation, and switching. It can thus play an important role in a future all in the fiber communication system.

The authors acknowledge the support of the U.S. Air Force Office of Scientific Research, the U.S. Office of Naval Research, and the Defense Advanced Research Projects Agency. J. Choi thanks the Department of Defense and the American Society for Engineering Education. J. M. Choi's e-mail address is johnchoi@ caltech.edu.

\section{References}

1. A. F. J. Levi, R. E. Slusher, S. L. McCall, J. L. Glass, S. J. Pearton, and R. A. Logan, "Directional light coupling from microdisk lasers," Appl. Phys. Lett. 62, 561-563 (1993). 
2. G. Griffel and S. Arnold, "Synthesis of variable optical filters using meso-optical ring resonator arrays," in Proceedings of the IEEE LEOS Annual Meeting, Vol. 2 (Institute of Electrical and Electronics Engineers, Piscataway, N.J., 1997), p. 165.

3. B. E. Little, S. T. Chu, H. A. Haus, J. Foresi, and J.-P. Laine, "Microring resonator channel dropping filters," J. Lightwave Technol. 15, 998-1005 (1997).

4. Y. Xu, Y. Li, R. K. Lee, and A. Yariv, "Scattering-theory analysis of waveguide-resonator coupling," Phys. Rev. E 62, 7389-7404 (2000).

5. A. Yariv, "Universal relations for coupling of optical power between microresonators and dielectric waveguides," Electron. Lett. 36, 321-322 (2000).
6. B. E. Little, H. A. Haus, J. S. Foresi, L. C. Kimerling, E. P. Ippen, and D. J. Ripin, "Wavelength switching and routing using absorption and resonance," IEEE Photon. Technol. Lett. 10, 816-818 (1998).

7. M. Cai and K. Vahala, "Highly efficient optical power transfer to whispering-gallery modes by use of a symmetrical dual-coupling configuration," Opt. Lett. 25, 260-262 (2000).

8. J. C. Slater, Microwave Electronics (Van Nostrand, New York, 1950). 